\title{
Regard neuf sur le monde rural dans tous ses états
}

\author{
Claire Binet et Prudence-Elise Breton ${ }^{1}$ \\ Secrétariat rural du Canada - Région du Québec
}

$\mathcal{E}$ n 1991, les États généraux du monde rural déclenchaient une mobilisation afin de revitaliser le tissu rural du Québec. Depuis, de nombreuses initiatives publiques et citoyennes s'appliquent à guider et à soutenir les communautés vers la prise en main de leur propre destinée pour que chacune puisse contribuer au mieux-être et à l'identité de l'ensemble de la société. Des défis de taille sont toutefois posés pour ces communautés : vieillissement de la population, mise en place d'un développement durable, globalisation des marchés, économie du savoir, etc. Dans ce nouveau contexte, l'état des connaissances sur les populations rurales devient une préoccupation accrue des acteurs du développement local et de l'aménagement du territoire.

\section{De nouvelles trouvailles pour des actions ciblées}

En matière statistique, les profils territoriaux actuellement disponibles sont teintés d'une " distorsion urbaine » : minoritaires et dispersées, les populations rurales y sont noyées dans des données d'ensemble et habituellement considérées comme une portion résiduelle aux caractéristiques imprécises. Pourtant, les zones rurales sont loin de constituer des espaces homogènes. Des recherches en cours au Secrétariat rural du Canada révèlent de surprenants constats ${ }^{2}$. À plusieurs égards, les différences à l'intérieur des milieux ruraux sont plus grandes que celles existant entre ceux-ci et les zones urbaines. Les premiers résultats indiquent également que certains écarts entre les milieux ruraux et urbains se maintiennent et s'accentuent même parfois.

Les efforts déployés pour ces recherches visent d'abord l'analyse de la répartition des populations rura- les pour cerner la complexité de leur structure spatiale, telle que décrite ci-avant. La variable population devient ainsi une trame de fond pour une caractérisation socio-économique avec des indicateurs de recensement ${ }^{3}$. Les résultats différenciés obtenus contribueront à une meilleure compréhension de problématiques rurales diverses. À terme, ces nouvelles connaissances aideront à cibler des interventions pour des segments spécifiques des populations rurales.

Au plan des résultats, cet article expose d'abord la structure spatiale des populations rurales du Québec et de cinq de ses régions, en incluant l'analyse des variations entre 1991 et 2001, selon les entités géographiques de la grille d'analyse. Pour illustrer les possibilités de ce nouvel outil, nous brossons ensuite un portrait de l'emploi dans le secteur manufacturier en 2001 et mettons ces constats en relation avec la dynamique démographique rurale observée.

\section{La structure spatiale changeante des populations rurales au Québec}

\section{Les zones à prédominance urbaine}

Établissons d'emblée que, en 2001, près de $80 \%$ de la population du Québec était rassemblée dans des zones à prédominance urbaine. Les 6 régions métropolitaines de recensement (RMR) regroupaient les deux tiers de la population, alors que les 25 agglomérations de recensement (AR) comptaient pour $12 \%$ (encadré $\mathrm{A}$ ). À cause de leur poids démographique élevé, les gouvernements s'en servent souvent comme référence pour des études soutenant l'élaboration de politiques et l'organisation territoriale. Signalons que ces zones 
à prédominance urbaine englobent aussi beaucoup de petites localités rurales aux caractéristiques socioéconomiques méconnues, d'où notre intérêt à les étudier. Dans l'évolution démographique récente, la croissance continue ${ }^{4}$ observée au Québec durant la décennie 1991-2001 n'a globalement profité qu'aux RMR : après diverses fluctuations, les AR et les zones à prédominance rurale ont enregistré un bilan négatif.

\section{Encadré A : Les zones à prédominance urbaine : les $A R$ et les $R M R^{5}$}

- Les agglomérations de recensement ou AR ont un noyau urbain (encadré C) qui contient entre 10000 et 100000 habitants (ex. : Granby, Drummondville, Val-d'Or, Sept-Îles).

- Le noyau urbain des régions métropolitaines de recensement, ou RMR, en contient 100000 ou plus (ex. : Montréal, Sherbrooke, Saguenay).

- Le lieu de travail a un effet structurant sur l'espace économique et les «bassins de vie ». À cet égard, le critère de navettage vers les noyaux urbains des AR et RMR est déterminant pour délimiter ces territoires.

- On y inclut les municipalités avoisinantes dont $50 \%$ ou plus de la population active occupée (travailleurs + chercheurs d'emploi) navette vers une AR ou une RMR.

\section{Les zones à prédominance rurale}

Quelque 1,5 million d'habitants vivaient dans les zones à prédominance rurale du Québec en 2001. Jusqu'à récemment, il n'y avait pas de concept géographique permettant une étude différenciée des populations rurales. Les déplacements multiples (personnes, biens et services) jouant un rôle croissant dans la structuration de l'espace (spécialisation des vocations, urbanisation diffuse), l'intensification des rapports ville-campagne a un effet indéniable sur la structure spatiale des populations (encadré B). Afin d'obtenir une nouvelle lecture des espaces ruraux, le premier niveau de la grille d'analyse a donc été élaboré en fonction de cette relation (graphique 1).

Cette grille d'analyse permet notamment d'observer que, entre 1991 et 2001, une concentration accrue de la population s'est effectuée dans les RMR et les ZIM fortes. Habituellement localisées autour des zones urbaines, ces dernières forment une couronne d'étalement urbain diffus (435 705 personnes en 2001, le quart des ZIM). Ce constat, de prime abord connu, comporte toutefois la nouveauté d'une proportion spécifique de navettage. Par contre, jamais auparavant un lien systématique n'avait encore été mesuré entre l'évolution des populations rurales et leur niveau d'intégration au marché du travail des zones urbaines. Ainsi, alors que les populations en ZIM fortes tiraient profit d'une proximité généralement plus grande des zones urbaines, le contingent des ZIM modérées (775 270 personnes, la moitié des ZIM) est demeuré plutôt stagnant. En outre, les ZIM faibles (273 785 personnes, moins d'un cinquième des ZIM) et les ZIM nulles (46530 personnes, part marginale des ZIM) ont subi un recul important depuis 1996.

\section{Encadré B : Les zones à prédominance rurale : les ZIM $^{6}$}

- La définition Régions rurales et petites villes (RRPV) ${ }^{7}$ énonce que les populations vivant en dehors des AR et des RMR sont considérées comme rurales.

- Le concept de Zone d'Influence Métropolitaine (ZIM) mesure le degré d'influence des zones urbaines sur les zones rurales à travers le niveau d'intégration de la main-d'œuvre rurale au marché de l'emploi AR/RMR.

- Ce degré est établi par le navettage total (quotidien, hebdomadaire ou moins fréquent) de la population active occupée vers une/plusieurs AR/RMR (adaptation du critère de délimitation des $A R / R M R)$.

- Le concept de ZIM intègre la notion de distance, l'ampleur du navettage diminuant généralement avec l'éloignement des zones urbaines. L'effet de distance peut toutefois être affecté par la capacité d'absorption du marché rural de l'emploi.

- Les municipalités sont réparties en quatre catégories de ZIM :

- ZIM fortes : $\geq 30 \%$ de navetteurs (ex. : SainteAnne-de-Beaupré, Métis-sur-Mer);

- ZIM modérées : 5\%-30\% de navetteurs (ex. : Maskinongé, Saint-Faustin);

- ZIM faibles : 0\%-5\% de navetteurs (ex. : LacMégantic, Paspébiac);

- ZIM nulles : $0 \%$ ou moins de 40 personnes dans la main-d'œuvre occupée (ex : Blanc-Sablon, Akwesasne).

Dans les cinq régions étudiées, les proportions de population en zone à prédominance rurale (ZIM) variaient passablement en 2001 :

- Montérégie : 19,6 \% (région centrale en croissance démographique); 
- Estrie : 38,3\% (région centrale en croissance démographique);

- Saguenay-Lac-Saint-Jean (SLSJ) : 28,1 \% (région périphérique en perte de population);
- Bas-Saint-Laurent (BSL) : 57 \% (région périphérique en perte de population);

- Abitibi-Témiscamingue (AT) : 39,5\% (région périphérique en perte de population).

\section{Graphique 1}

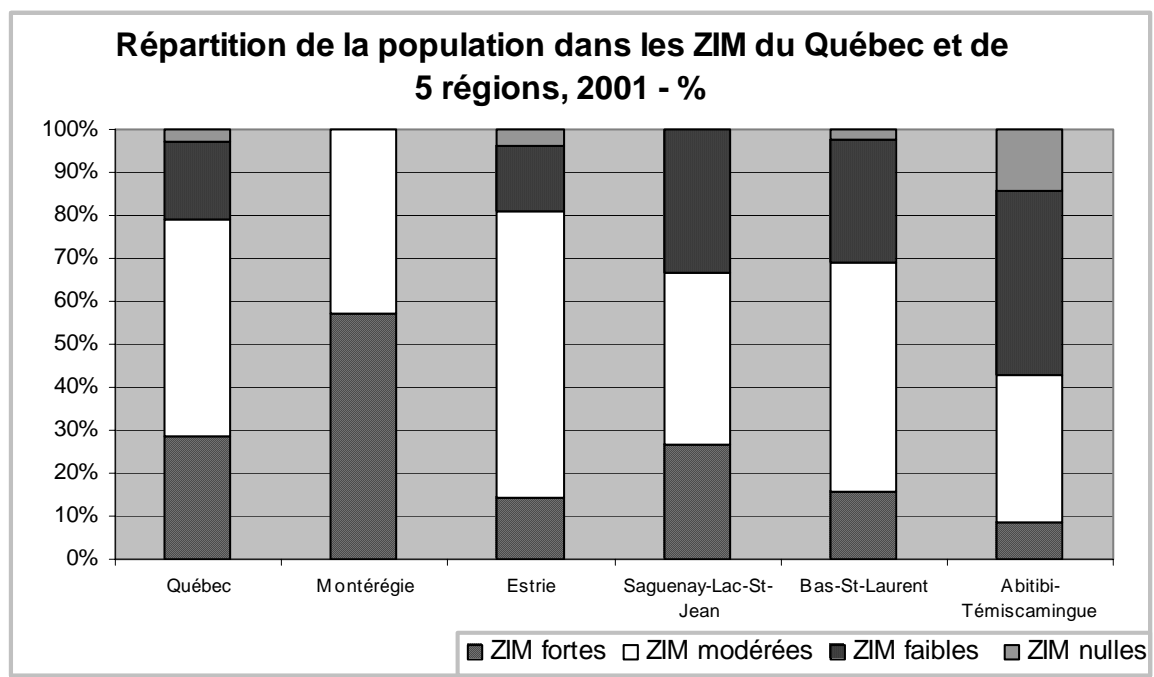

Plusieurs autres éléments de dissemblance, voire de divergence, apparaissent entre le Québec et les régions étudiées (graphique 1). Ces différences reflètent entre autres leur situation géographique (centrale/ périphérique) et leur évolution spécifique. En voici les faits saillants :

- le Bas-Saint-Laurent et l'Abitibi-Témiscamingue n'ont pas de RMR, mais on y trouve trois AR; pour sa part, la Montérégie en compte six;

- cette dernière région n'a pas de ZIM faibles; en outre, elle ne comporte pas de ZIM nulles, tout comme le Saguenay-Lac-Saint-Jean;

- la part de chaque type de ZIM par rapport au total des zones rurales peut varier; par ex. : ZIM modérées en Estrie : $2 / 3$ du total des ZIM; ZIM fortes en Abitibi-Témiscamingue : $1 / 12$ du total des ZIM; ZIM faibles de la même région : $2 / 5$ du total des ZIM;

- les AR des régions étudiées n'ont pas enregistré de baisse entre 1991 et 2001 : stagnation au BasSaint-Laurent, hausse en Montérégie, en Estrie et en Abitibi-Témiscamingue (baisse globale mais hausse considérée seulement à cause de la $\mathrm{AR}$ d'Amos ajoutée en 2001);
- des augmentations ont été recensées en ZIM modérées pour la Montérégie et l'Abitibi-Témiscamingue et en ZIM faibles pour l'Estrie et le Saguenay-Lac-Saint-Jean;

- les ZIM fortes ont subi une diminution en AbitibiTémiscamingue, mais elles ont stagné depuis 1996 en Montérégie;

- la RMR du Saguenay-Lac-Saint-Jean a perdu de la population durant la décennie 1991-2001.

\section{Un destin différencié pour les villes et les petites localités rurales}

Les zones à prédominance rurale et urbaine ci-dessus décrites contiennent à la fois des petites municipalités rurales et des villes de tailles variées (encadré C).

\section{Encadré $C$ : Les aires rurales et urbaines du recensement}

Une AIRE RURALE a un noyau de population :

- de moins de 1000 habitants OU

- dont la densité est inférieure à 400 habitants $/ \mathrm{km}^{2}$.

- Ce sont des petites localités situées en ZIM (ex. : Péribonka, Val-Alain);

- ou certaines petites localités en banlieue rurale AR/RMR (ex. : l'Île d'Orléans, Hatley). 
Une AIRE URBAINE a noyau urbain :

- qui compte 1000 habitants ou plus ET

- dont la densité est supérieure ou égale à 400 habi$\operatorname{tants} / \mathrm{km}^{2}$.

- Ce sont des petites villes situées en ZIM (ex. : Amqui, Louiseville);

- ou des villes-centres des AR/RMR (ex. : Montréal, Drummondville, Saint-Georges);

- ou des noyaux urbains secondaires AR/RMR (ex. : Laval, Lévis, Beloeil).

N.B. La population des aires rurales/urbaines des municipalités de grande taille peut être comptée séparément (ex : Amos et Mont-Saint-Hilaire : $80 \%$ urbain et $20 \%$ rural).

L'analyse rurale croisée combine les trois types de concept présentés jusqu'ici (AR/RMR, ZIM, aires rurale/urbaine). Elle permet d'analyser ces populations rurales et urbaines à une échelle plus détaillée, dans le contexte spécifique des zones à prédominance rurale ou urbaine où elles sont localisées (encadré D).

\section{Encadré D : L'analyse rurale croisée (combinaison des concepts) $^{8}$}

- La grille d'analyse combine les AR/RMR et les 4 ZIM ( $1^{\text {er }}$ niveau d'analyse) auxquelles s'ajoutent les aires rurales/urbaines ( $2 \mathrm{e}$ niveau d'analyse).

- L'extension maximale de la grille d'analyse crée 18 entités par l'intégration de ces deux niveaux : 6 zones ( 2 urbaines et 4 rurales), avec chacune leurs composantes rurale/urbaine. Selon l'une ou l'autre des définitions, RRPV ou recensement, 16 de ces 18 entités ont une population en totalité ou en partie rurale.
- La valeur ajoutée de cette méthode d'analyse permet notamment de cerner certaines situations et dynamiques internes aux zones (petites villes ZIM, banlieues rurales $\mathrm{AR} / \mathrm{RMR})$.

- Population rurale totale $=$ total ZIM + banlieues rurales AR/RMR. C'est grossièrement la population couverte par la Politique nationale de la ruralité du gouvernement du Québec.

Ainsi, les banlieues rurales constituent une « ceinture rurale » entre, d'une part, les aires urbaines des AR/ RMR et, d'autre part, les localités rurales et les petites villes des ZIM. Cette couronne périurbaine varie grandement en importance d'une région à l'autre (graphique 2).

En 2001, la plus grande partie des populations ZIM du Québec vivait dans des petites localités en aire rurale; environ le tiers des résidents des zones rurales était toutefois globalement regroupé dans les aires urbaines (petites villes) des ZIM (graphique 2). La dispersion en aire rurale était très élevée dans les ZIM nulles et la plus basse proportion en aire rurale était observée dans les ZIM faibles (tableau 1). Par ailleurs, l'examen des variations à l'intérieur des ZIM pour la période 1991-2001 révèle une diminution notable dans les petites municipalités des aires rurales et une tendance générale à l'urbanisation. Ce constat met en évidence le renforcement des petites villes et une nette coupure avec les petites localités rurales en déclin (tableau 2).

\section{Graphique 2}

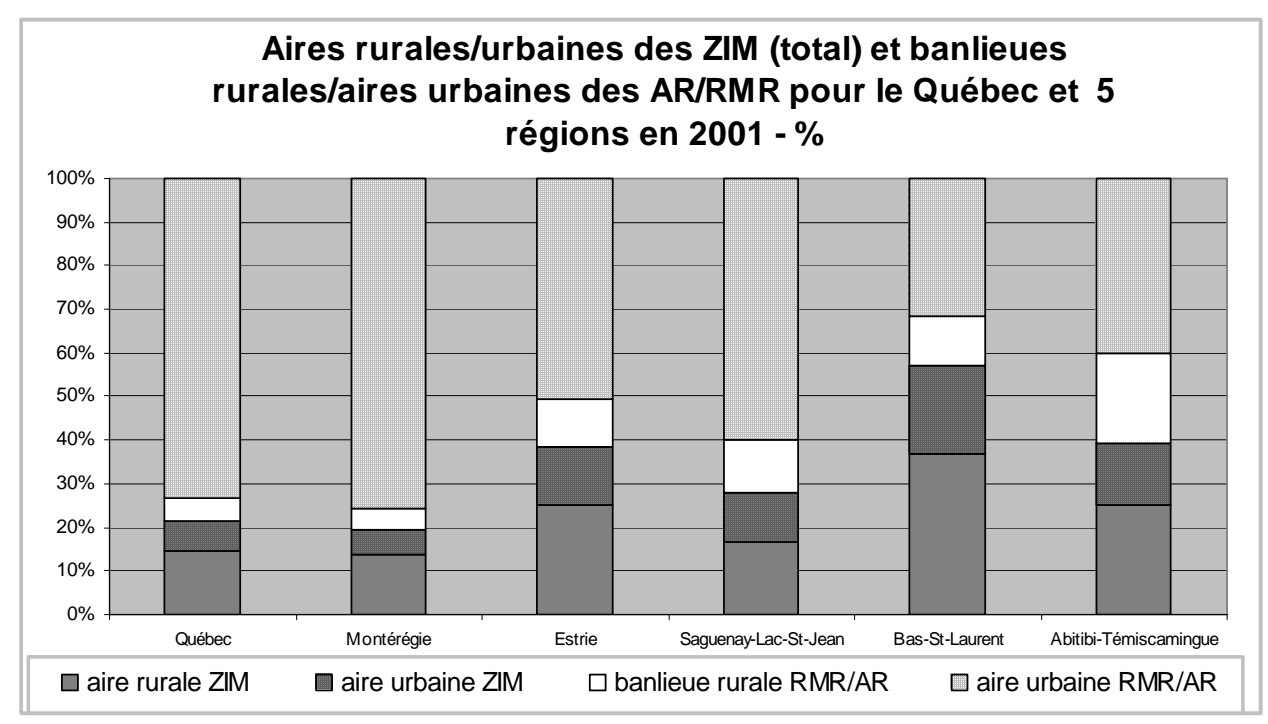


Tableau 1 - Population des ZIM en aire rurale et des AR/RMR en banlieue rurale, 2001 (\%)

\begin{tabular}{lcccccc} 
& $\begin{array}{c}\text { ZIM } \\
\text { fortes }\end{array}$ & $\begin{array}{c}\text { ZIM } \\
\text { modérées }\end{array}$ & $\begin{array}{c}\text { ZIM } \\
\text { faibles }\end{array}$ & $\begin{array}{c}\text { ZIM } \\
\text { nulles }\end{array}$ & AR & RMR \\
\hline Québec & $76 \%$ & $64 \%$ & $60 \%$ & $91 \%$ & $16 \%$ & $5 \%$ \\
Montérégie & $72 \%$ & $67 \%$ & n.a. & n.a. & $9 \%$ & $5 \%$ \\
Estrie & $100 \%$ & $59 \%$ & $58 \%$ & $100 \%$ & $22 \%$ & $17 \%$ \\
Saguenay-Lac-Saint-Jean & $67 \%$ & $72 \%$ & $35 \%$ & n.a. & $9 \%$ & $19 \%$ \\
Bas-Saint-Laurent & $94 \%$ & $59 \%$ & $56 \%$ & $100 \%$ & $27 \%$ & n.a. \\
Abitibi-Témiscamingue & $100 \%$ & $60 \%$ & $48 \%$ & $100 \%$ & $34 \%$ & n.a.
\end{tabular}

Source : Statistique Canada, Recensement 2001, compilation spéciale.

\section{Tableau 2 - Tendances de l'évolution 1991-2001 à l'intérieur des ZIM et AR/RMR du Québec, comparaison avec les tendances dans 5 régions}

\begin{tabular}{|c|c|c|}
\hline & $\begin{array}{c}\text { ZIM : Baisse de population dans les aires rurales et } \\
\text { renforcement des aires urbaines }\end{array}$ & $\begin{array}{l}\text { AR/RMR : Urbanisation dans les } \\
\text { banlieues rurales (empiètement?) }\end{array}$ \\
\hline Québec & $\begin{array}{l}\text { - Stagnation en aire rurale des ZIM fortes } \\
\text { - Baisse depuis } 1996 \text { en aire rurale des ZIM modérées, } \\
\text { faibles et nulles } \\
\text { - Hausse en aire urbaine, sauf en ZIM nulles }\end{array}$ & $\begin{array}{l}\text { - Baisse après } 1996 \text { dans les RMR } \\
\text { - Baisse dans les AR }\end{array}$ \\
\hline Montérégie & $\begin{array}{l}\text { Non : } \\
\text { - Baisse en aire rurale des ZIM fortes } \\
\text { - Stagnation en aire rurale des ZIM modérées }\end{array}$ & Oui \\
\hline Estrie & $\begin{array}{l}\text { En partie : } \\
\text { - Hausse en aire rurale des ZIM fortes }\end{array}$ & $\begin{array}{l}\text { Non : } \\
\text { - Stable dans la RMR et la AR }\end{array}$ \\
\hline $\begin{array}{l}\text { Saguenay-Lac- } \\
\text { Saint-Jean }\end{array}$ & $\begin{array}{l}\text { En partie : } \\
\text { - Baisse en aire rurale des ZIM fortes }\end{array}$ & $\begin{array}{l}\text { En partie : } \\
\text { - Baisse continue dans la RMR } \\
\text { - Chute de plus de } 50 \% \text { dans les } 2 \text { AR }\end{array}$ \\
\hline Bas-Saint-Laurent & $\begin{array}{l}\text { En partie : } \\
\text { - Hausse en aire rurale des ZIM fortes }\end{array}$ & $\begin{array}{l}\text { En partie : } \\
\text { - Baisse dans les } 3 \text { AR depuis } 1996\end{array}$ \\
\hline $\begin{array}{l}\text { Abitibi- } \\
\text { Témiscamingue }\end{array}$ & $\begin{array}{l}\text { En partie : } \\
\text { - Baisse en aire rurale des ZIM fortes } \\
\text { - Hausse en aire urbaine des ZIM modérées } \\
\text { - Disparition des aires urbaines en ZIM nulles }\end{array}$ & $\begin{array}{l}\text { Non : } \\
\text { - Augmentation à cause de la création de la } \\
\text { AR d'AR d'Amos (recensement 2001) }\end{array}$ \\
\hline
\end{tabular}

Source : Statistique Canada, Recensement 2001, compilation spéciale

Pour leur part, les banlieues rurales des AR/RMR regroupaient plus du quart de la population des petites localités rurale au Québec en 2001 (graphique 3A). Soulignons que les caractéristiques socio-économiques de ces 375000 ruraux travaillant majoritairement « en ville» sont parfois contrastées par rap- port à celles des urbains. D'ailleurs, le reclassement en aire urbaine d'une partie des résidents des banlieues rurales a fait diminuer leur nombre entre 1991 et 2001. De plus, leur poids dans les AR et RMR est limité, voire marginalisé (graphique $3 \mathrm{~B}$, tableaux 1 et 2). 
La situation des régions étudiées se présente parfois de manière assez différente de celle du Québec. Soulignons ici quelques tendances régionales :

- Dans les ZIM fortes de l'Estrie, du Bas-SaintLaurent et de l'Abitibi-Témiscamingue, la présence de résidents uniquement dans des petites localités rurales dénote une tendance à un étalement urbain diffus, ce phénomène ayant été amplifié par une hausse démographique en Estrie et au BasSaint-Laurent.

- En Montérégie, le recul démographique des aires rurales en ZIM fortes et leur stagnation en ZIM modérées dans un contexte général de croissance démographique semble refléter une urbanisation très active.

- Au Bas-Saint-Laurent et au Saguenay-Lac-SaintJean, les aires urbaines des ZIM faibles avaient

\section{Graphique 3A}

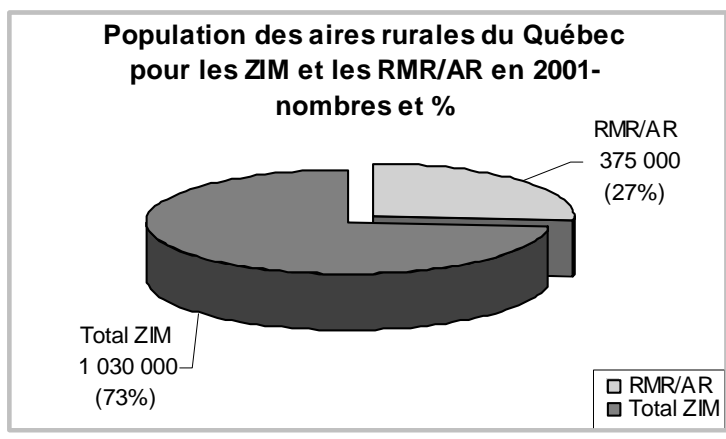

\section{L'urbanisation en marche ...}

À maints égards, la démographie influe sur la planification des équipements et les affectations du sol (résidences, commerces, services, etc.). Inversement, les décisions d'aménagement peuvent aussi avoir un impact sur les données du recensement. Ainsi, le déclin des populations dans les banlieues rurales AR/RMR et, jusqu'à un certain point, dans plusieurs aires rurales des ZIM, n'est pas causé par des départs mais bien plutôt par l'expansion urbaine. L'empiétement du nouveau bâti sur les banlieues rurales entraine le reclassement en aire urbaine de nombreux résidents ruraux (atteinte des seuils de taille et de densité d'aire urbaine). L'augmentation de la population des aires urbaines n'est donc pas toujours synonyme de croissance démographique; elle peut aussi résulter d'un étalement urbain causé par la relocalisation de respectivement atteint $45 \%$ et $65 \%$, ce qui met en évidence une importante composante urbaine en zone rurale.

- Dans les trois régions qui ont des ZIM nulles (Estrie, BSL et AT), les habitants sont totalement dispersés dans des petites localités des aires rurales.

Les banlieues rurales du Saguenay-Lac-Saint-Jean et de l'Abitibi-Témiscamingue englobaient plus de $40 \%$ de la population des petites localités de ces deux régions en 2001; de plus, les aires rurales des ZIM fortes y ont subi un recul depuis 1991.

Ces constats démontrent que l'étalement urbain serait davantage circonscrit à l'intérieur des zones urbaines qu'en périphérie des $A R$, intégrant ainsi les populations rurales à la vie urbaine.

\section{Graphique 3B}

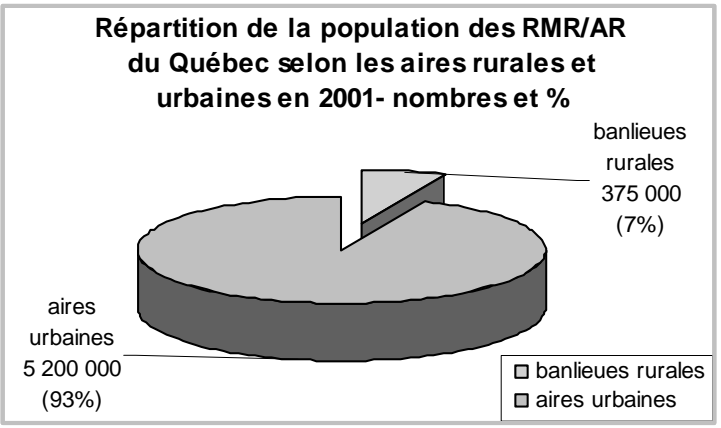

résidents dans des nouveaux quartiers en périphérie. Inévitablement, ces facteurs contribuent à l'altération graduelle de ces espaces ruraux (rapports des individus avec leur environnement physique et social, mode de vie et culture).

Le classement en aire rurale/urbaine reflète la réalité du terrain, mais le degré d'interrelation rurale-urbaine se mesure par le niveau de ZIM ou l'intégration à une AR/RMR. Avec le temps, la hausse du taux de navettage d'une municipalité peut entraîner un reclassement (autre ZIM ou AR/RMR). Si le noyau urbain d'une petite ville atteint 10000 habitants, notamment à la suite de l'extension de son bâti, on crée une AR. Ce fut le cas d'Amos pour le recensement de 2001, malgré que la population totale de la nouvelle AR ait diminué entre 1996 et $2001{ }^{9}$ : on a soustrait la ville d'Amos aux ZIM faibles tout en englobant 10 muni- 
cipalités rurales (en ZIM modérées ou faibles) comptant au moins $50 \%$ de navetteurs vers Amos. Enfin, d'autres changements surviennent à la suite de fusions : ainsi, en 2002, six municipalités des banlieues rurales de la $\mathrm{AR}$ et quelques localités en ZIM fortes se sont fusionnées à la ville de Rouyn-Noranda, ce qui fera augmenter le poids des banlieues rurales de l'AT lors du recensement de 2006.

Qu'il s'agisse d'une expansion urbaine concrète sur le terrain, d'un reclassement statistique ou d'une réorganisation territoriale, ces situations témoignent toutes d'une intégration croissante des populations rurales aux zones urbaines. La méthode d'analyse présentée ici permet de mesurer le degré d'intégration ruraleurbaine (ZIM, AR/RMR), tout en captant les changements sur le terrain (aires rurales/urbaines). La structure spatiale des populations rurales devient alors la couche d'information de base sur laquelle on peut apposer différentes caractéristiques. L'indicateur du pourcentage d'emplois dans le secteur manufacturier illustrera cette démarche.

\section{Les emplois dans le secteur manufacturier}

\section{La situation générale au Québec}

En 2001, les emplois du secteur manufacturier étaient, en nombres absolus, trois fois plus importants dans les zones urbaines du Québec que dans les zones rurales, soit 474740 contre 165930 , ce qui n'a rien d'étonnant étant donné la concentration massive de la population dans les zones urbaines. Cependant, $22,6 \%$ des travailleurs des ZIM se retrouvaient dans ce secteur, alors qu'on en recensait seulement 16,3\% dans les AR/RMR. Ce fait illustre a priori le rôle majeur de ce type d'emploi en milieu rural : d'autres travaux nous ont d'ailleurs déjà permis de constater que le secteur tertiaire occupait généralement une plus grande place en milieu urbain, alors que le secteur primaire était davantage présent en milieu rural.

En ce qui a trait aux parts d'emploi dans le secteur manufacturier, on notait une différence de six points en faveur des AR, comparativement aux RMR, mais l'écart était plus marqué entre les catégories de zones rurales, soit 10,5 points. Dans ces dernières, les proportions tendaient généralement à diminuer en fonction de la baisse de l'influence urbaine : un taux assez élevé en ZIM fortes (21,4\%), un pic en ZIM modérées $(25,1 \%)$, puis un pourcentage moindre dans les ZIM faibles $(18,4 \%)$, avec finalement le plus bas niveau en ZIM nulles (14,6\%). Les petites villes des ZIM fortes et modérées affichaient les proportions les plus hautes de tout le Québec, tandis que celles des ZIM nulles se trouvaient à l'autre extrême (graphique 4). Les aires urbaines des AR affichaient des proportions un peu plus élevées que les banlieues rurales, alors qu'à l'intérieur des RMR, les proportions étaient à peu près égales. Selon les travaux en cours, les pourcentages similaires des banlieues rurales RMR et des petites localités rurales des ZIM nulles n'ont pas la même portée, la proportion du secteur tertiaire étant plus élevée en banlieue rurale RMR.

\section{Graphique 4}

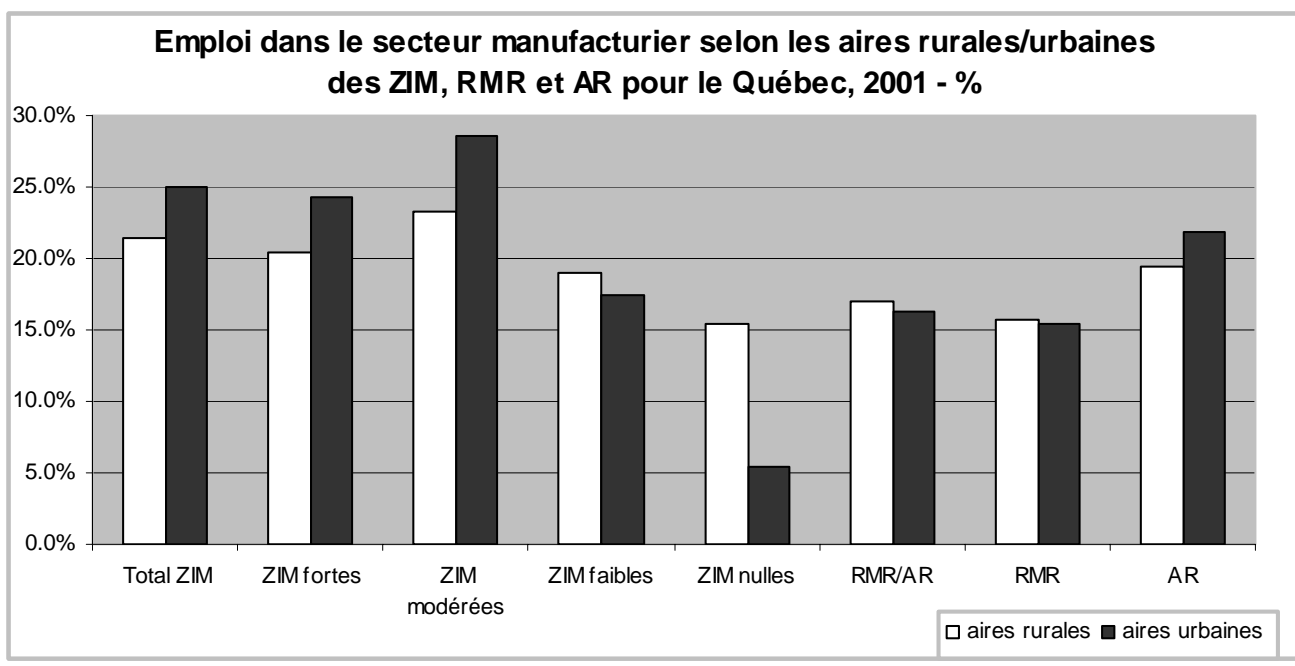


La différenciation observée ici entre les divers milieux ruraux offre une nouvelle perspective : la superposition de cette répartition des emplois manufacturiers sur les constats précédents au sujet des populations rurales révèle que les proportions d'emploi dans ce secteur étaient moins importantes dans les milieux en recul démographique, soit les ZIM faibles et nulles ainsi que dans les banlieues rurales des AR/RMR. Des analyses préliminaires démontrent la présence importante du secteur tertiaire dans les zones urbaines, mais elles confirment aussi que les emplois du secteur primaire étaient plus présents dans les ZIM faibles et nulles. Signalons que les variations de population entre 1991 et 2001 s'inscrivaient dans une période où se sont amorcés d'importants changements structurels, en plus de l'avènement de la globalisation des marchés mondiaux. Ainsi, les emplois ont fortement chuté dans le secteur primaire, avec des effets d'entraînement marqués sur l'industrie de première transformation ${ }^{10}$. Cet impact a pu être plus marqué dans les ZIM faibles et nulles. S'il advenait que le secteur manufacturier soit plus fortement affecté par des délocalisations liées à la mondialisation, l'importante place du secteur manufacturier dans les milieux ruraux pourrait faire en sorte que ceux-ci souffriraient davantage.

\section{La situation dans les régions étudiées}

Sous l'angle des régions visées par cette étude, leur situation spécifique ressemblait peu au profil d'ensemble pour le Québec. Pour faciliter une lecture comparée, nous avons utilisé les quotients de localisation $(\mathrm{QL}){ }^{11}$, en plus des pourcentages. De prime abord, les régions centrales (Montérégie, Estrie) avaient en 2001 une plus grande part d'emplois manufacturiers que les régions périphériques (SLSJ, BSL, AT). Signalons que, dans les cinq régions, les proportions étaient plus élevées dans les ZIM que dans les AR/RMR; mais aucune région ne présentait une répartition conforme aux constats notés au Québec pour les ZIM. De plus, les variations internes étaient plus ou moins grandes : pour tous les types de comparaison intra-régionales, l'Estrie et le SaguenayLac-Saint-Jean se retrouvaient aux antipodes, la première étant championne des écarts, la seconde présentant des contrastes minimes et une relative homogénéité.

Étonnamment, les régions étudiées affichaient les plus hauts pourcentages en ZIM faibles ou nulles, alors que les proportions y étaient les plus basses à l'échelle du Québec. La plus élevée atteignait 45,1\%, dans les petites villes des ZIM faibles de l'Estrie. Cette dernière a enregistré une croissance continue des emplois pour ce secteur depuis le milieu des années 1990 de même qu'une hausse démographique, principalement en ZIM faibles. Illustrant l'écart marqué entre les régions, le taux le plus bas de l'Estrie $(24,1 \%$, en banlieue rurale RMR) demeurait plus élevé que le plus haut des 18 entités de chacune des trois régions périphériques $(21,4 \%$ en ZIM faibles rurales, AT), qui ont toutes perdu de la population entre 1991 et 2001.

La concentration des emplois manufacturiers dans la première transformation était élevée en 2001 dans ces trois régions (environ $80 \%$ en AT, $75 \%$ au SLSJ et $55 \%$ au BSL, contre $30 \%-35 \%$ pour l'ensemble du Québec). Le lien était assez net avec les niveaux d'emploi primaire qui, selon nos travaux, étaient aussi plus élevés dans les ZIM faibles et nulles de ces régions. À cause de cette double concentration, ces régions perdent plus d'emplois en cette période de grands changements.

\section{Des recherches plus poussées et d'autres angles d'approches}

De tels résultats étant difficiles à interpréter en soi, il faudrait examiner parallèlement les autres secteurs d'emploi ainsi que certaines variables pouvant apporter un éclairage plus large (par ex. : taux d'activité, paiements de transfert). Par ailleurs, l'état actuel de nos analyses ne permet pas d'isoler la part des emplois manufacturiers liée à la première transformation des ressources. De plus, nous n'avons pas encore pu mesurer les changements survenus entre 1991 et 2001.

Ces constats sommaires soulignent la nécessité de se pencher sur la question des niveaux de transformation dans le secteur manufacturier, principalement en lien avec un secteur primaire omniprésent en milieu rural et ce, particulièrement dans les régions périphériques. Les emplois en deuxième et troisième transformation créent une valeur ajoutée dont les retombées pourraient être déterminantes pour la diversification économique rurale et pour la qualité de vie des ruraux, si on sait tirer profit des opportunités. Ne pourrait-on pas mesurer la progression de ce développement selon les zones rurales, les petites localités, les petites villes et même les banlieues rurales ? 
Par ailleurs, une exploration plus détaillée des différents sous-secteurs de l'industrie manufacturière (papier, textile, aliments, etc.) aiderait à mieux comprendre la structure industrielle des espaces ruraux, notamment dans les ZIM fortes et modérées: les parts d'emploi observées dans ce secteur étaient plutôt élevées dans les deux régions du sud du Québec mais très modestes dans les trois régions périphériques. Beaucoup d'interrogations surgissent. Quels sous-secteurs manufacturiers sont stables ou en croissance? Quels sous-secteurs sont en déclin ou en difficulté ? Dans quel type de milieu rural observe-t-on ces phénomènes?

Au-delà de la conjoncture économique mondiale, les politiques gouvernementales et les orientations régionales en matière de développement et d'aménagement du territoire influencent aussi l'emploi dans les secteurs primaire et manufacturier. Voici quelques exemples de situation :

- secteur forestier : conflit du bois d'œuvre, pratiques d'aménagement de la forêt, mode de gestion et de transformation des ressources (collectif, petite entreprise, grand capital), baisse du niveau des coupes de bois;

- secteur agroalimentaire: production de masse pour les nécessités de base, diversification et produits de niche à valeur ajoutée, problèmes de cohabitation pour certaines productions;

- rareté ou difficulté d'accès à certaines autres ressources (pêches et mines) : éloignement de certains bassins miniers, gestion des ressources (ex. : moratoire dans les pêcheries).

\section{Un nouveau chantier rural en perspective}

L'analyse rurale croisée est assurément un outil d'analyse des populations rurales générant des informations inédites. Déjà, les constats présentés ici ont identifié des segments spécifiques de population rurale, tout en soulignant l'importance structurante des activités manufacturières pour l'économie de ces milieux. Ils suscitent aussi des questions incitant à poursuivre les travaux. En matière de planification régionale, ces nouvelles connaissances invitent à une réflexion sur des enjeux liés aux problématiques soulevées et ce, dans la perspective d'un développement durable tenant compte des particularités variées des communautés rurales.
Une évidence s'impose : le recul démographique des petites localités est une tendance lourde qui ne date pas d'hier. Malgré certains succès, un renversement global du processus est peu probable; il serait irréaliste de chercher à rétablir les niveaux de population d'antan. Tout comme la planète entière, le monde rural est en mutation. Les années futures donneront lieu à une stabilisation des populations rurales autour d'une reconfiguration des systèmes de production, notamment par l'économie du savoir et la globalisation des marchés, entraînant d'inévitables effets sur la gouvernance, l'aménagement du territoire et l'organisation des services ${ }^{12}$.

Devant de telles perspectives, le recours à ce nouvel instrument d'analyse spatiale, et ce pour des situations similaires dans des régions différentes, pourrait favoriser l'établissement de diagnostics communs mais nuancés en fonction de petites entités jusqu'ici un peu négligées au plan statistique. L'élaboration de solutions adaptées à des besoins spécifiques en serait d'autant facilitée. En outre, ces nouvelles connaissances permettraient de développer un système de veille sans pareil pour des communautés vulnérables.

Avec le temps, le plus grand mérite de cette nouvelle lecture spatiale pourrait-il être d'inverser la perspective d'analyse du territoire ? De la vision urbanocentrée habituelle des $\mathrm{RMR} / \mathrm{AR}$ versus la périphérie rurale, on passera à une lecture faisant place aux espaces peu peuplés mais regorgeant parfois de potentiels méconnus. Au grand pique-nique du développement rural, les populations des grandes villes s'entasseront désormais dans seulement deux des dix-huit cases composant la grande nappe à carreaux de cette grille d'analyse : on pourra alors enfin observer le déploiement rural dans toute sa diversité (et tous ses états!).

\section{Notes et références}

1 Géographe au parcours atypique, Claire Binet est analyste régionale pour le Québec et l'Ontario au Secrétariat rural du Canada. Préoccupée par le territoire, elle a œuvré depuis une vingtaine d'années en analyse et recherche, en aménagement du territoire et en formation dans plusieurs sphères, notamment dans le secteur agricole. PrudenceElise Breton est diplômée en service social. Particulièrement intéressée par l'organisation communautaire durable, elle complète un stage de neuf mois en recherche au Secrétariat rural du Canada.

2 Un projet pan-canadien (un profil pour chaque province/ territoire) examine vingt indicateurs des recensements 
1991-1996-2001 à travers une analyse comparative des zones rurales et urbaines de même qu'une analyse différenciée des zones rurales à l'aide du concept de ZIM élaboré par Statistique Canada.

(A venir en 2005, voir http://rural.gc.ca).

3 D'autres recherches sur l'évolution territoriale des populations démontrent l'importance d'une trame spatiale de base pour la variable population, notamment Gauthier, M.-J. (2004), « Profils territoriaux de l'évolution de la population du Saguenay-Lac-Saint-Jean, 1951-2001 », Organisations et territoires, vol. $13, \mathrm{n}^{\circ} 2$. La description de la typologie des variations d'effectifs de la population et les résultats pour cette région rejoignent plusieurs aspects de la grille d'analyse spatiale présentée ici de même que nos résultats spécifiques pour ladite région.

4

Les données employées pour ces recherches correspondent à l'échantillonnage de $20 \%$ du recensement, tel que compilé pour les caractéristiques de la population, ce qui ne comprend pas les résidents des institutions.

5 Les concepts et la méthodologie présentés dans les encadrés viennent de Statistique Canada. Voir notamment le Dictionnaire du recensement :

www12.statcan.ca/francais/census01/Products/Reference/d ict/index_f.htm

6 McNiven, C., H. Puderer et D. Janes (2000). Zones d'influence des régions métropolitaines de recensement et des agglomérations de recensement (ZIM) : une description de la méthodologie, série de documents de travail de la géographie, $\mathrm{n}^{\circ} 2000-2$, Statistique Canada

(www.statcan.ca/cgi-bin/downpub/listpub_f.cgi?catno= 92F0138MIF).

7 Beshri, R., R. Bollman, H. Clemenson et V. Du Plessis (2001). « Définitions de rural », Bulletin d’analyse régions rurales et petites villes du Canada, vol. $3, \mathrm{n}^{\circ} 3$, Statistique Canada

(http://www.statcan.ca:8096/bsolc/francais/bsolc?catno $=21$ -006-X\&CHROPG=1).

8 Mendelson, R. (2001). Les structures géographiques comme variables de recensement: se servir de la géographie pour analyser les phénomènes sociaux et économiques, série de documents de travail de la géographie, n 2001-1, Statistique Canada.

9 Statistique Canada, Profil des communautés, www12.statcan.ca/francais/profil01/PlaceSearchForm1_F. $\mathrm{cfm}$.

10 Nos propos concernant l'emploi manufacturier dans la première transformation ainsi que sur la répartition et l'évolution des emplois dans les secteurs primaire et manufacturier réfèrent notamment à : Polèse, $M$. et R. Shearmur (2005). La dynamique spatiale de l'économie canadienne et l'avenir des régions périphériques du Québec, LASER, INRS-UCS, présentation à l'ACFAS.

11 Le QL établit un rapport entre deux \% (QL = \% du Québec divisé par le $\%$ de la région). $\mathrm{Un} \mathrm{QL}=1$ indique un niveau égal à celui du Québec; un $\mathrm{QL}<1$ reflète une proportion inférieure et un QL > 1 une part plus grande.

12 Polèse M. et R.. Shearmur avec la collaboration de P.-M. Desjardins et M. Johnson (2002), La périphérie face à l'économie du savoir: la dynamique spatiale de l'économie canadienne et l'avenir des régions non métropolitaines du Québec et des provinces de l'Atlantique, Montréal, INRSUCS et l'Institut canadien de recherche sur le développement régional, $237 \mathrm{p}$.

(www.inrs-ucs.uquebec.ca/default.asp? $p=$ res). 